\title{
Geometrical and Experimental Conditions for the Homogeneous and Inhomogeneous Flows of the Polyethylene Oxide Solution in the Cylinder of Couette
}

\author{
Ngargoto Ngarmoundou ${ }^{1,2}$, El Hadji Oumar Gueye ${ }^{1}$, Mahamat Barka ${ }^{3}$, \\ Aboubaker Chedikh Beye ${ }^{1, *}$ \\ ${ }^{1}$ Group Laboratories of Physics Solid State and Sciences of Materials, Département of Physics, Faculty of Science and Technics, University \\ Cheikh Anta Diop of Dakar, Dakar, Senegal \\ ${ }^{2}$ Department of Mechanic, Sciences and Technologies Faculty, Polytechnic University of Mongo, Mongo, Chad \\ ${ }^{3}$ Department of Physical Sciences, Faculty of Exact and Applied Sciences, University of N'Djamena, N'Djaména, Chad
}

Email address:

nngarmoundou@yahoo.fr (N. Ngarmoundou), elhadjioumar.gueye@gmail.com (El H. O. Gueye), acbeye@gmail.com (A. C. Beye)

${ }^{*}$ Corresponding author

\section{To cite this article:}

Ngargoto Ngarmoundou, El Hadji Oumar Gueye, Mahamat Barka, Aboubaker Chedikh Beye. Geometrical and Experimental Conditions for the Homogeneous and Inhomogeneous Flows of the Polyethylene Oxide Solution in the Cylinder of Couette. American Journal of Modern Physics. Vol. 7, No. 2, 2018, pp. 75-86. doi: 10.11648/j.ajmp.20180702.13

Received: January 9, 2018; Accepted: January 19, 2018; Published: February 23, 2018

\begin{abstract}
A simple method for characterizing the flow curve of a polymer solution (PEO) in shear in Couette geometry is developed. It consists in considering a priori the fluid in Newtonian flow with the assumptions related to the influence of the rheological and geometrical parameters, then to evaluate the shearing speed characteristic of this partially and/or totally sheared material. The results obtained by the numerical simulations in 2D and in 3D in this flow configuration allow to find a heterogeneity of rheological behavior related to the properties of the fluid on the one hand and on the other hand, to size the Couette geometry while fixing explicitly the experimental conditions according to whether the fluid is Newtonian or not Newtonian.
\end{abstract}

Keywords: Couette Flow, Rheological Behavior, Shear Stress, Shear Rat

\section{Introduction}

Couette coaxial cylinder geometry is widely used to evaluate the flow velocity profiles of materials. In such a geometry, the shear rate is heterogeneous and depends on the property of the fluid and the width of the air gap between the coaxial cylinders. The techniques for calculating the shear rate at the inner cylinder wall are generally based on simplifying assumptions related to the size of the air gap and the choice a priori of the rheological behavior law of the sheared fluid.. The main problem then consists in establishing a reliable relationship between the torque and the rotational speed of the geometry on the one hand and on the other hand, between the stress and a shear rate characteristic of the shear of the fluid.

In this work a method will be used to evaluate the PEO flow curves developed by numerical simulation in the centered cylindrical Couette geometry. It involves locally considering the flowing fluid as a Newtonian fluid, and evaluating the characteristic shear rate of the generated fluid that is partially and/or completely sheared. These deployed velocity curves generally depend on the experimental rotation speed and the radius of the virtual cylinder internally bounded by $R_{1}$ and externally by $R_{2}$ fixed by the initial hypothesis for a Newtonian fluid. Adjustment of the virtual radius makes it possible to reach the linear velocity curves for precise rotational speed values, and to perfectly fix the values of the radius $R_{1}$ and $R_{2}$ of the Couette cylinder for a Newtonian fluid. In order to validate our procedure, we considered experimental data from the literature, studying in particular the influence of the behavior of the fluid, as well as the size of the geometry.

Nomenclature 
$r$ virtual cylinder position $(\mathrm{mm})$

$r_{c}$ flow and rest area interface $(\mathrm{mm})$

$R_{i}$ radius of the virtual cylinder $(\mathrm{mm})$

$R_{1}$ radius of the inner cylinder ( $\left.\mathrm{mm}\right)$

$R_{2}$ radius of the outer cylinder ( $\left.\mathrm{mm}\right)$

$\mathrm{K}$ consistency $\left(\mathrm{N} \cdot \mathrm{sec}^{2} / \mathrm{m}^{2}\right)$

$\mathrm{n}$ flow index

$V_{Z}$ axial component of the speed $(\mathrm{mm} / \mathrm{s})$

$V_{r}$ radial component of velocity $(\mathrm{mm} / \mathrm{s})$

$V_{(\theta, r)}$ tangential component of velocity $(\mathrm{mm} / \mathrm{s})$

$V_{0}$ maximum tangential velocity expressed as a function of angular velocity $(\mathrm{mm} / \mathrm{s})$

$\mathrm{M}$ couple $(\mathrm{Nm})$

$R_{e}$ Reynolds number

$K_{N G A}$ Period of flow of the fluid in the air-gap

Greek letters

-

$\gamma$ shear rate $\left(s^{-1}\right)$

- $\gamma_{c}$ critical shear rate $\left(s^{-1}\right)$

$\gamma_{c}$

$\tau$ shear stress $\mathrm{Pa}$

$\tau_{c}$ Critical shear stress $(\mathrm{Pa})$

$\omega$ rotation speed $(\mathrm{rd} . / \mathrm{s})$

$\omega_{c}$ critical rotation speed ( $\left.\mathrm{rd} . / \mathrm{s}\right)$

$\sigma_{i}$ shear stress at the virtual cylinder $(\mathrm{Pa})$

$\eta$ viscosity (Pa.s)

$\mu$ kinematic viscosity $\left(\mathrm{m}^{2} / \mathrm{s}\right)$

$\rho$ density $\left(\mathrm{g} / \mathrm{cm}^{-3}\right)$

Acronyms

PEO polyethylene oxide

Indices

$\mathrm{C}$ critical point

1-2 index at point 1 , respectively 2 .

i virtual point index

\section{Principle of the Method}

The sample of material used in this study is a solution containing polyethylene-oxide (PEO) of molar mass $10^{6}$ $\mathrm{gmol}^{-1}$ and of concentration $1,8 \% \mathrm{wt}$. The choice is related to the geometry of Couette with shearing imposed (i.e. man can measures the couple on the interior cylinder after having imposed its number of revolutions $\omega=2 \pi \mathrm{N} \mathrm{rd}$./s. It is of coaxial cylinders type to broad air-gap in front of the dimension of the microstructure to avoid the effect of size which makes the surface smooth of the cylinder $\mathrm{R}_{1}$ sensitive to the slip from where its coating sometimes with the Emery fabric). Shearing in the geometry is homogeneous, which can guarantee that the material with the same structure in all the air-gap. The two coaxial cylinders are of interior radius $\mathrm{R}_{1}=$ $13,375 \mathrm{~mm}$ is revolving at an angular velocity from $(0,002$ to $0,9 \mathrm{rd} . / \mathrm{s})$, which gives the low speeds of shearing ranging between 0.0026 to $1,20 \mathrm{~cm} / \mathrm{s}$ and the macroscopic rates of shearing from $\left(0,2\right.$ to $\left.96 \mathrm{~S}^{-1}\right)$. The $\mathrm{R} 1$ cylinder height $\mathrm{h}=$ $40,12 \mathrm{~mm}$ ensures a sufficient contact to increase the couple. The external radius $\mathrm{R}_{2}=13,5 \mathrm{~mm}$ is fixed, the curve $\mathrm{R} 1 / \mathrm{R} 2=$ 0,99 . The digital simulations of the profiles speeds are operated with the MATLABR2008b software. The conversion of the macroscopic data into law of behavior then requires an analysis adapted for the identification of the material [1]. Man considers in more our fluid incompressible, laminar, permanent fluid and the inertias are in all negligible points with respect to the forces of viscosity. Under these conditions, we proceed to the research of the profile speed as follows:

Profile speed for a Newtonian fluid in geometry of Cylindrical Couette

Whatever the fluid in laminar flow in the cylindrical geometry of Couette, the rate of shearing $\dot{\gamma}$ in cylindrical coordinates is expressed by the equation (1) and depends on the tangential component speed $\mathrm{V}_{(\theta, \mathrm{r})}(\mathrm{r})$ from which it derives [2], [3].

$$
\dot{\gamma}=\frac{\partial V_{(\theta, r)}(r)}{\partial r}-\frac{V_{(\theta, r)}(r)}{r}=r \frac{\partial}{\partial r}\left(\frac{V_{(\theta, r)}}{r}\right)
$$

The shear stress $\tau(\mathrm{r})$ being exerted on the internal cylinder is directly related to the couple, $\mathrm{M}$, independently of the nature of the fluid [4].

$$
\tau(r)=\frac{M}{2 \pi h r^{2}}
$$

The equation of Navier Stockes, in the case of a Newtonien fluid becomes then:

$$
\frac{\partial}{\partial r}\left(r^{3} \frac{\partial}{\partial r}\left(\frac{V_{(\theta, r)}}{r}\right)\right)=0
$$

By taking account of the boundary conditions and nonslip, the profile tangential speed of a Newtonien fluid in a cylindrical geometry of Couette is given by the equation 4 and the equation 5:

$$
V_{(\theta, r)}(r)=\frac{\omega R_{1}^{2}}{R_{2}^{2}-R_{1}^{2}}\left(-r+\frac{R_{2}^{2}}{r}\right)
$$

The equation (5) reformulates the equation (4) by taking account of the assumption that the inertias are negligible compared to the forces of viscosity and so that, the rate of flow takes into account the property of the solution related to the number of Reynold $R_{e}=\left(\omega R_{1} e\right) / \mu$ with $\mu=\eta / \rho$, via a constant. The geometry of the device of Couette has the internal radius $\mathrm{R}_{1}$ and external radius $\mathrm{R}_{2}$ [5].

$$
\left\{\begin{array}{l}
V_{(\omega, r)}\left(R_{e}, r\right)=R_{e} K_{N G A}\left(-r+\frac{R_{2}^{2}}{r}\right) \\
\text { with }: \\
K_{N G A}=\frac{\mu R_{1}}{\left(R_{2}+R_{1}\right) e^{2}}
\end{array}\right.
$$

$K_{N G A}\left(s^{-1}\right)$ is the period of flow of the fluid in the air-gap. The rate of shearing which results from this is given by the 
expression of the equation 6 :

$$
\dot{\gamma}(r)=\frac{2 \omega R_{1}^{2} R_{2}^{2}}{R_{2}^{2}-R_{1}^{2}} \frac{1}{r^{2}}
$$

One obtains the expressions of the rate of shearing and of the constraint which strongly depend on the ray of the virtual cylinder in the air-gap and because of this dependence in rheometry, the constraints and rate of shearing are measured at the same place. For a Newtonian fluid $\tau(\mathrm{r})=\eta \dot{\gamma} \mathrm{r})$ but in practice, the commercial rheometers indicate average values of the constraint and local rate of shearing in the air-gap.

$$
\prec \sigma \succ=\frac{R_{1}^{2}+R_{2}^{2}}{4 \pi h R_{1}^{2} R_{2}^{2}} M-\prec \dot{\gamma} \succ=\frac{R_{1}^{2}+R_{2}^{2}}{R_{2}^{2}-R_{1}^{2}}
$$

If the fluid is not Newtonien, man can expect a difference between the real law of behavior of material $\tau=\mathrm{f}(\gamma)$ and that given by the rheometer $\langle\tau\rangle=\mathrm{f}(<\dot{\gamma}>)$. Any time when the cell used presents a small air-gap, i.e. when

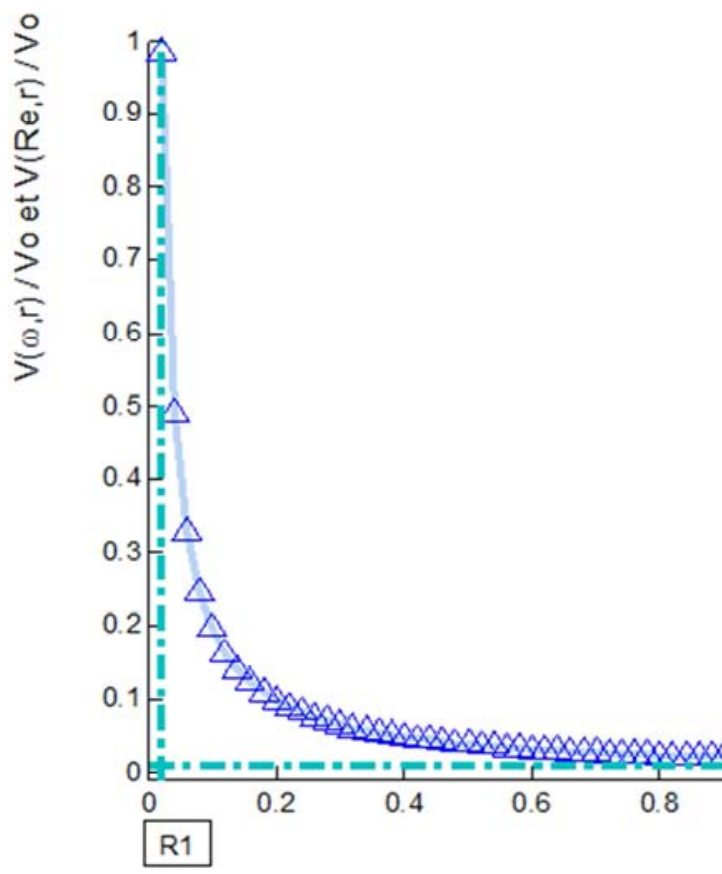

$\left(R_{2}-R_{1}\right)<<R_{1}$, the curve of flow becomes linear and man can confuse the macroscopic law of the rheological behavior given by the rheometer with the majority of the local rheological behaviors no Newtonian. We will primarily make the characteristic in this work by using a rheometer with broad air-gap to identify the variable and real behavior sheared fluid.

\section{Numerical Applications}

Digital simulations in $2 d$ and $3 d$ of the profiles speed a) Digital simulation in $2 d$

The digital simulation of the profile speed in $2 d$ of the figure 1 and the figure 2 is adimensionned. Profiles obtained following a digital simulation with MATLABR2008b at an angular velocity $\omega=0,9 \mathrm{rd} . / \mathrm{s}$ for the first figure and with a variation angular velocity of $(0,02$ to 0,9$) \mathrm{rd}$./s for the second. They translate the behavior of this structure in the geometry of Couette (coexistence of two areas different and the existence from an unstable zone in the curve of flow of material) [5], [6], [7], [8].

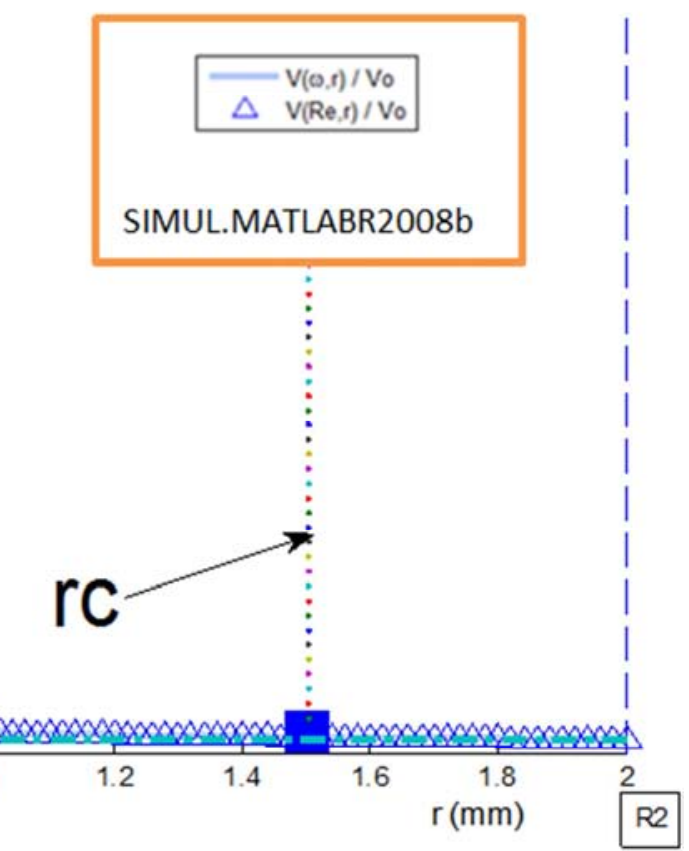

Figure 1. Local 2D-sized airspeed profile in the air gap of a Couette cylinder, a) Medialization according to the initial theoretical law of the fluid velocity of $V(\omega, r) / V_{0}$ (continuous line), b) according to the modified theoretical law of the velocity of the fluid $V(R e, r) / V_{0}$ (triangular marks). $r_{c}$ marks the interface between the solid zone and the liquid zone for a flow in the geometry. The variation of the shear rate with the velocity profile from $\gamma 2$ to $\gamma 1$ is observed before falling into the blocked zone [5].

b) The values criticize number of revolutions speed

In order to show all influence them angular velocity and number of Reynold on the modeling of the profiles speed which represents the flow of our fluid in the cylinder of Couette, we still refer to the figure 2: We note that, for number of revolutions $\omega>\omega_{\mathrm{c}}=0,8 \mathrm{rd} . / \mathrm{s}$, which corresponds to the Reynolds number $\mathrm{R}_{\mathrm{e}}>\mathrm{R}_{\mathrm{ec}}=54.10^{-4}$, the profiles fallback speeds $V(\omega, r) /$ Vo values not nul in the air-gap have everywhere and remain very close from/to each other. What is not the case for the lower number of revolutions speed, precisely for $\omega<\omega_{\mathrm{c}}=0,6 \mathrm{rd}$./s, which corresponds to the Reynolds number $\mathrm{R}_{\mathrm{e}}<\mathrm{R}_{\mathrm{ec}}=3.10^{-3}$, shearing is located more and more near the interior cylinder with the reduction this number of revolutions. This influence number of revolutions speed and the Reynolds number was raised in the results obtained in IRM in work of [9], [10]. These various phenomena which intervene during the flow in particular: the localization of shearing and the band of shearing were treated in work of [1]. 


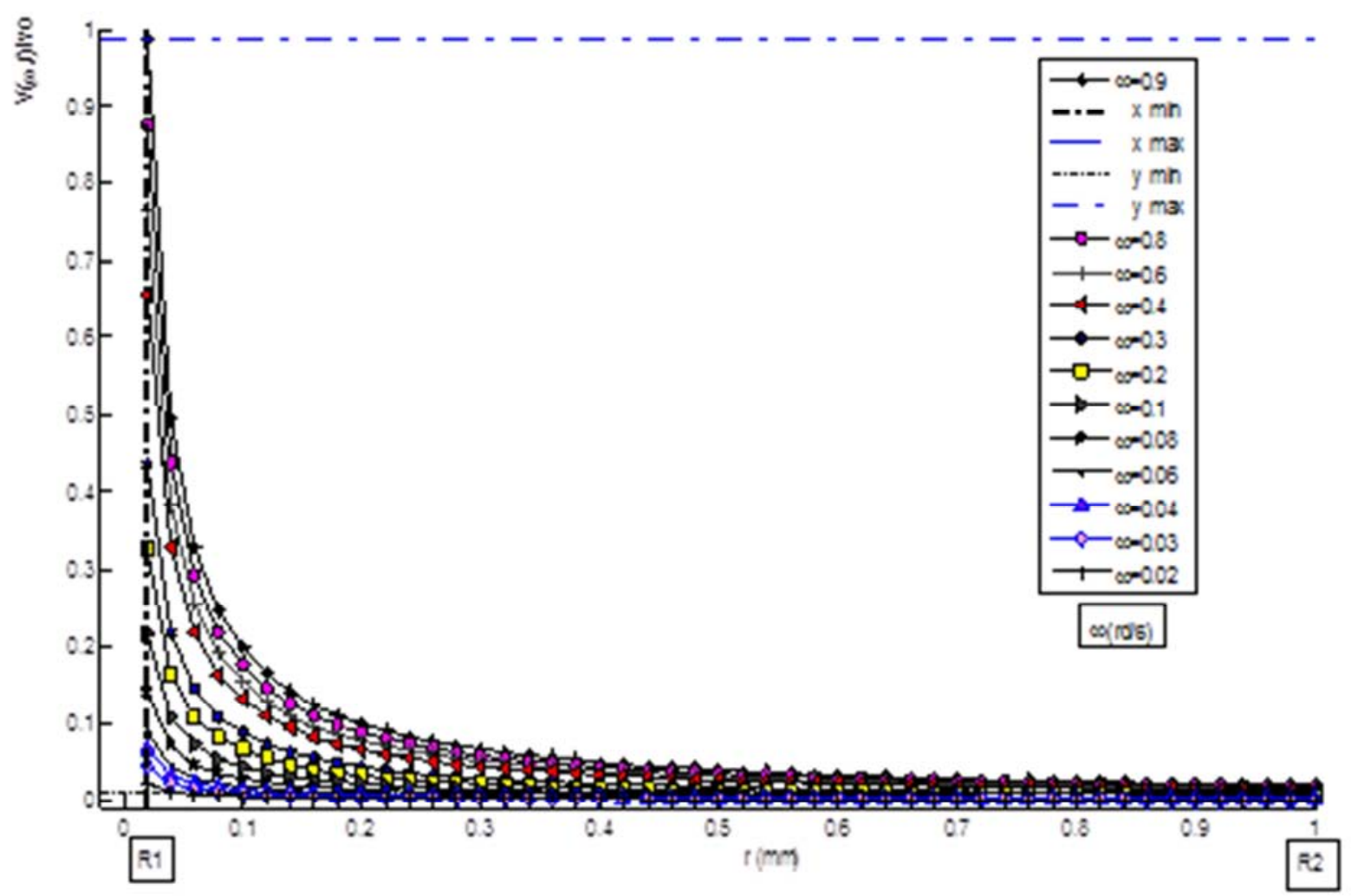

Figure 2. Local 2D-dimensional velocity profiles in the air gap of a Couette cylinder with the variation of the experimental parameters: $V(\omega, r) / V_{0}$ for different rotational angular velocities (0.02 to $0,9 \mathrm{rd}$. / s) imposed on the inner cylinder [5].

\section{c) Digital simulation in $3 d$}

The digital simulation in 3D has as a principal interest to see the profiles speed in space mode in order to graphically clear the various zones of the profile speed and as well the conditions of appearance of inhomogenous flows in stationary regime. It is significant to understand that the local rheological model used must obey in certain conditions so that blocked areas and areas in flow can coexist figure 3 a). Indeed in this case the same material, subjected to the same shear stress $\tau_{c}$, must be simultaneously in two states distinct from deformation translates by the figures into $2 \mathrm{D}$ and $3 \mathrm{D}$. A mathematical condition which allows this coexistence is the existence of an unstable zone in the curve of flow of the material [11], [12], [13], caused by a decrease of the rheological law in a certain range of rate of shearing. But as the portion of instability is not a general case of the curves of flow; the case of the ship's boys, how the coexistence of bands at various rates of shearing is it possible? [14].

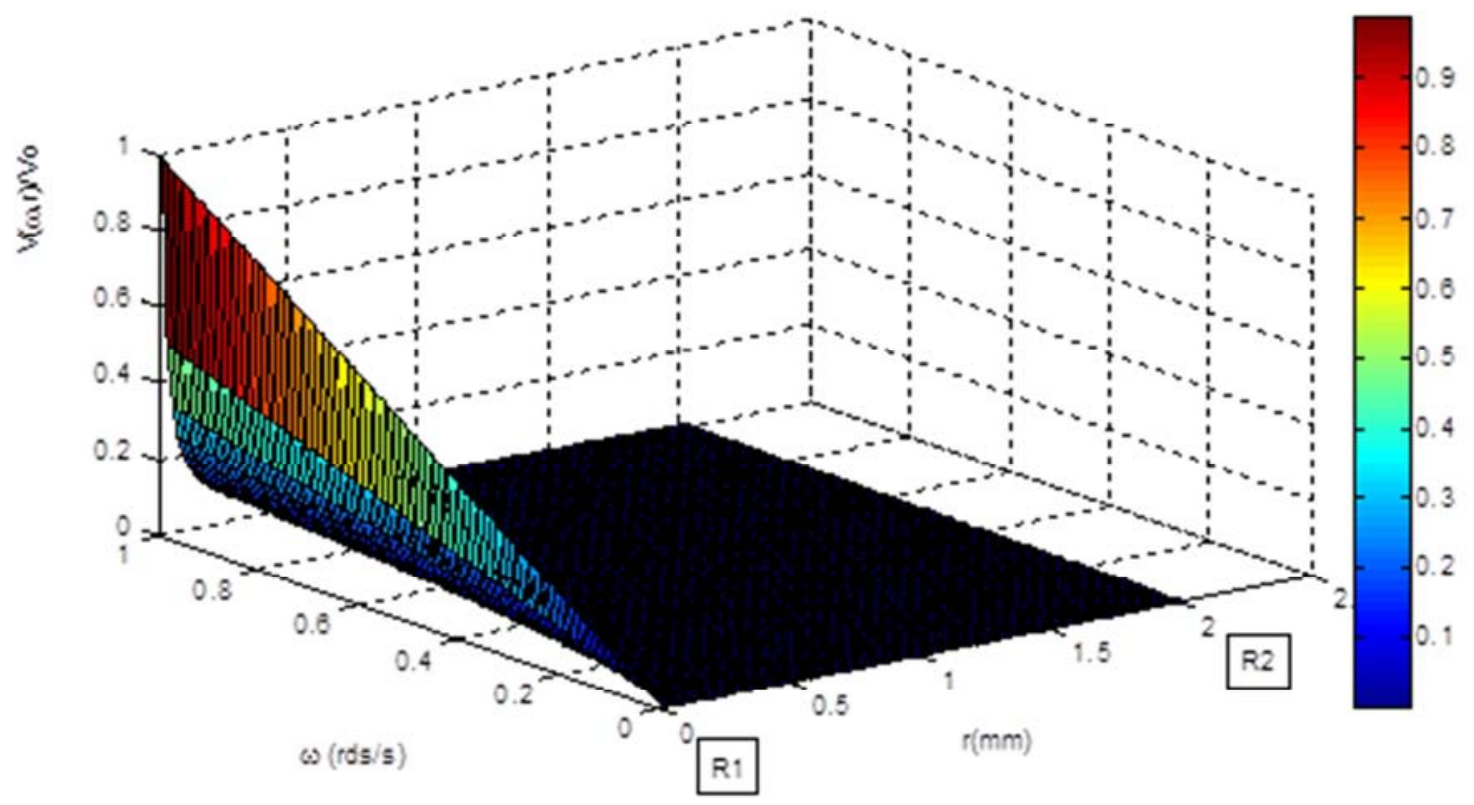




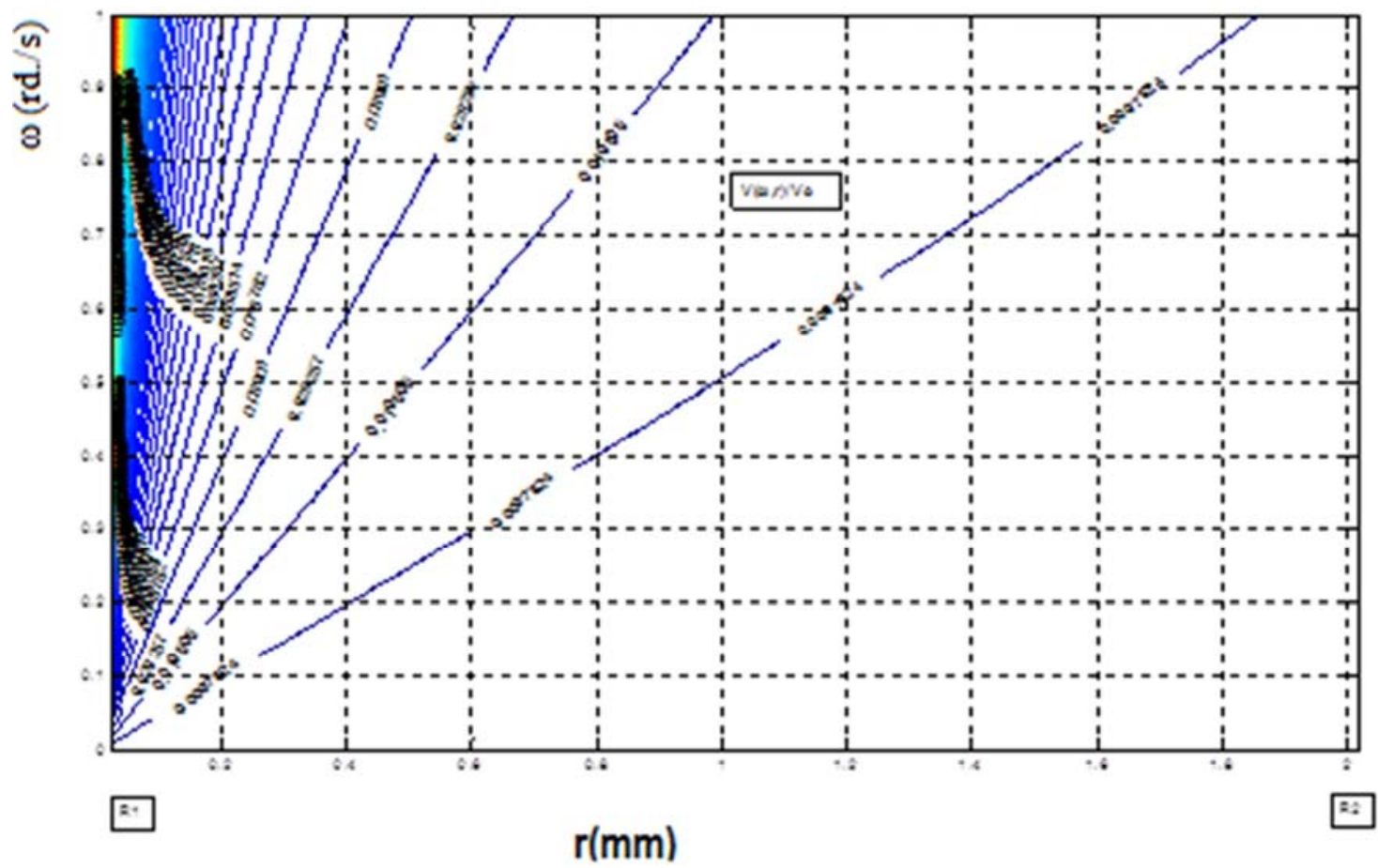

b)

Figure 3. a) 3D-dimensioned velocity profiles $V(\omega, r)) / V_{0}$ or $V(R e, r) / V_{0}$ showing the blocked regions and the flowing regions. The color bar informs us about the evolution of the magnitude of the speed profiles as a function of the parameters on which they depend in the air gap. Thus the high shear level of 0.65 to 1 at high speed in a restricted air gap develops high shear rates with very low viscosity. This viscosity increases gradually with the reduction of the shear rate from 0.65 to 0.35 and then from 0.35 to 0 with the increase of the air gap: we pass from the flow zone to the blocked zone. b) Profiles of the same velocities in the plane $(\omega, r)$ showing a localization around the cylinder $R_{1}[5]$.

\section{d) Observation and analyzes}

Simulations in $2 \mathrm{~d}$ and $3 \mathrm{~d}$ show (dependence $\eta(\dot{\gamma})$ ) nonlinear curve of flow already implemented by work of [5], [15], [16]. The fluid seems a fluid with threshold of constraint. Many authors specified this concept of constraint threshold [17], [18], [19], [20]. These materials can be solid under a certain level of constraint, and thus to have a certain arrangement in which the elements are blocked, and liquids beyond a certain constraint, and thus to have an indifferent arrangement and variable components. These fluids undergo a soft transition from the solid mode to the liquid mode around a given constraint, which means that its curve of flow ( $\tau$ vs $\dot{\gamma}$ ) presents, in a representation logarithmic curve a plate with the weak gradients speed. Its apparent viscosity thus tends towards the infinite one when $\dot{\gamma} \rightarrow 0$.

The two curves in $2 \mathrm{~d}$ and $3 \mathrm{~d}$ have also a discontinuity on the slopes of the profiles speeds in this geometry of Couette. This situation translates the heterogeneity of the rate of shearing and also of the constraints in the fluid. The profiles speeds have each one an almost constant slope $\dot{\gamma}_{2}$ at a significant distance, and they fall to $\dot{\gamma} 1$ as in the figure 3 then tend towards zero and remains around this value for larger distances from the interior cylinder. They correspond thus to the profils speeds obtained for a flow in giant micelle shearing [21], in a viscometer of Couette by laser approach of velocimetry (PIV: Velocimetry by Image of Particles) and nuclear velocimetry (IRM:Imagery by Magnetic Resonance), for granular materials [9], the polymer suspensions [10], and more generally for the complex fluids [22], [23], [11]. The model of the fluid under such a flow is known, it is about a fluid with rhéofluidifiant threshold of constraint and thus not Newtonien. The real law of behavior of material depends on the rate of shearing $\tau=\mathrm{f}(\dot{\gamma})$, different from that given by the rheometer $\langle\tau\rangle=\mathrm{f}(<\dot{\gamma}>)$. However, while taking account of our procedure of work which consists in varying the air-gap as envisaged at the end of paragraph 1.1, will manage to us to dimension the geometry for the flow of the homogeneous and nonhomogeneous structure and to fix the experimental conditions at the same time.

\section{Dimensioning of the Geometry with Coaxial Cylinders of Couette}

With an aim of seeking the conditions of a homogeneous flow characterized by the linearity of these curves and as well, the conditions under which the real law of behavior of material is identified with that measured by the rheometer envisaged at the end of paragraph 1.1, we simultaneously carry out digital simulations of the curves of flow in $2 \mathrm{~d}$ and $3 \mathrm{~d}$, by varying the number of revolutions speed and the space of shearing which is the air-gap. Our curves have identical results in $2 \mathrm{~d}$ and $3 \mathrm{~d}$ by sweeping all the number of revolutions in a way increasing from (0 to 1$) \mathrm{rd}$./s for a fixed value of the fictitious cylinder (r) included understood enters 
$\mathrm{R}_{1}=13,375 \mathrm{~mm}$ and $\mathrm{R}_{2}=13,5 \mathrm{~mm}$. The figure 4 and figure 7 represent the extreme stages, then the figure 5 and figure 6 the intermediate stages of the process. By varying four times in a decreasing way the fictitious ray ( $r$ ) since first value $0,125 \mathrm{~mm}, 0,04 \mathrm{~mm}, 0,028 \mathrm{~mm}$ and $0,022 \mathrm{~mm}$ respectively according to the figure 4 , figure 5 , figure 6 , figure 7 (at the same time for the figures in $2 \mathrm{~d}$ and $3 \mathrm{~d}$ ) and, by sweeping each time all the values number of revolutions in a way increasing from ( 0 to 1$) \mathrm{rd} . / \mathrm{s}$, the flow of the fluid is partially homogeneous until the number of revolutions threshold $\omega_{c}$ $\mathrm{rd} . / \mathrm{s}$ which depend on the value of the air-gap. The report is the same one for all the values of the fictitious ray up to $0,022 \mathrm{~mm}$ or the flow is completely homogeneous.

a) The angular velocity varies from ( 0 to $1 \mathrm{rd} . / \mathrm{s})$ in a going air-gap from $(0,02$ to 0,125$) \mathrm{mm} ; \omega_{\mathrm{c}} \geq 0,03 \mathrm{rd}$./ $\mathrm{s}$

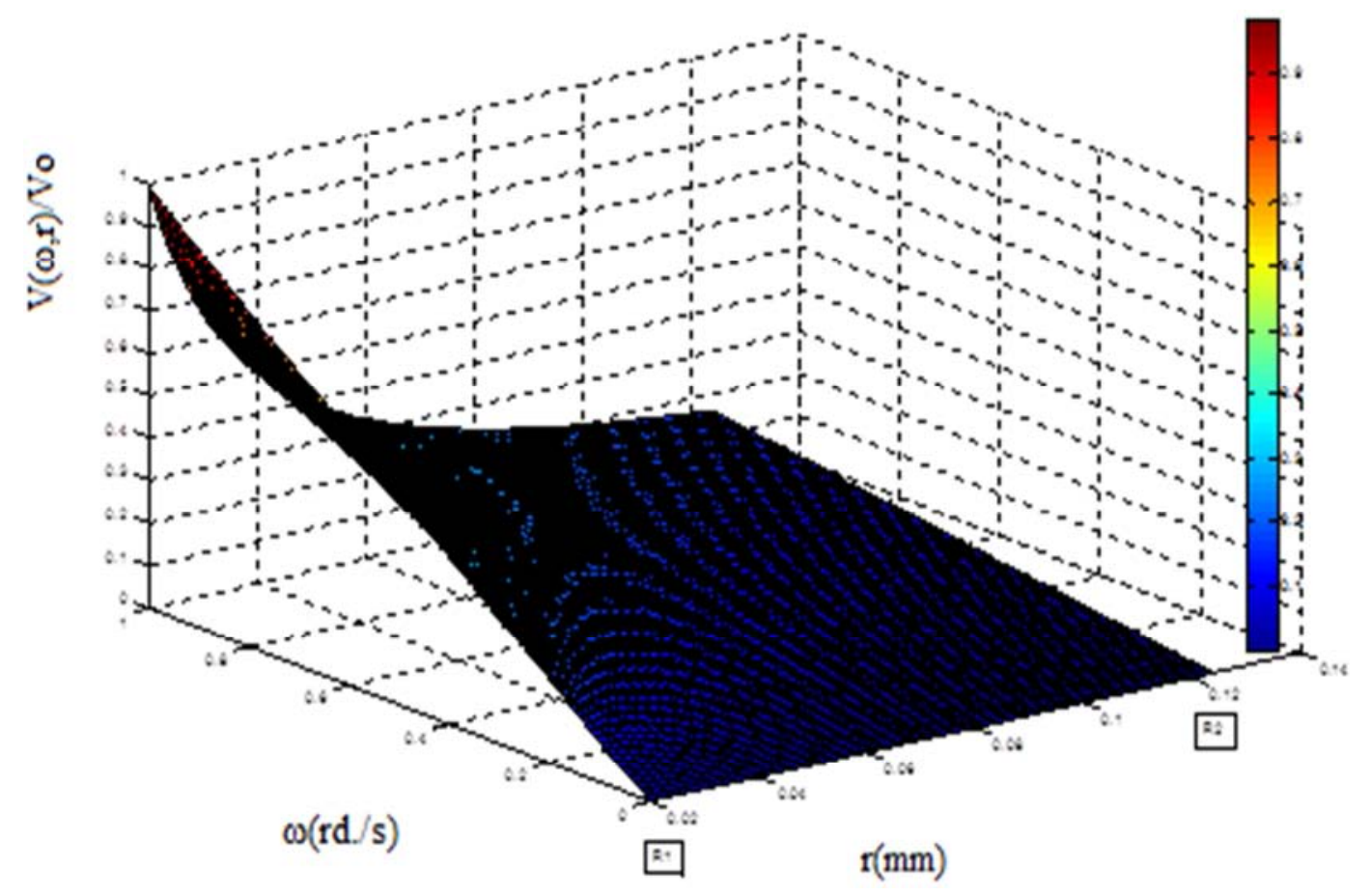

a)

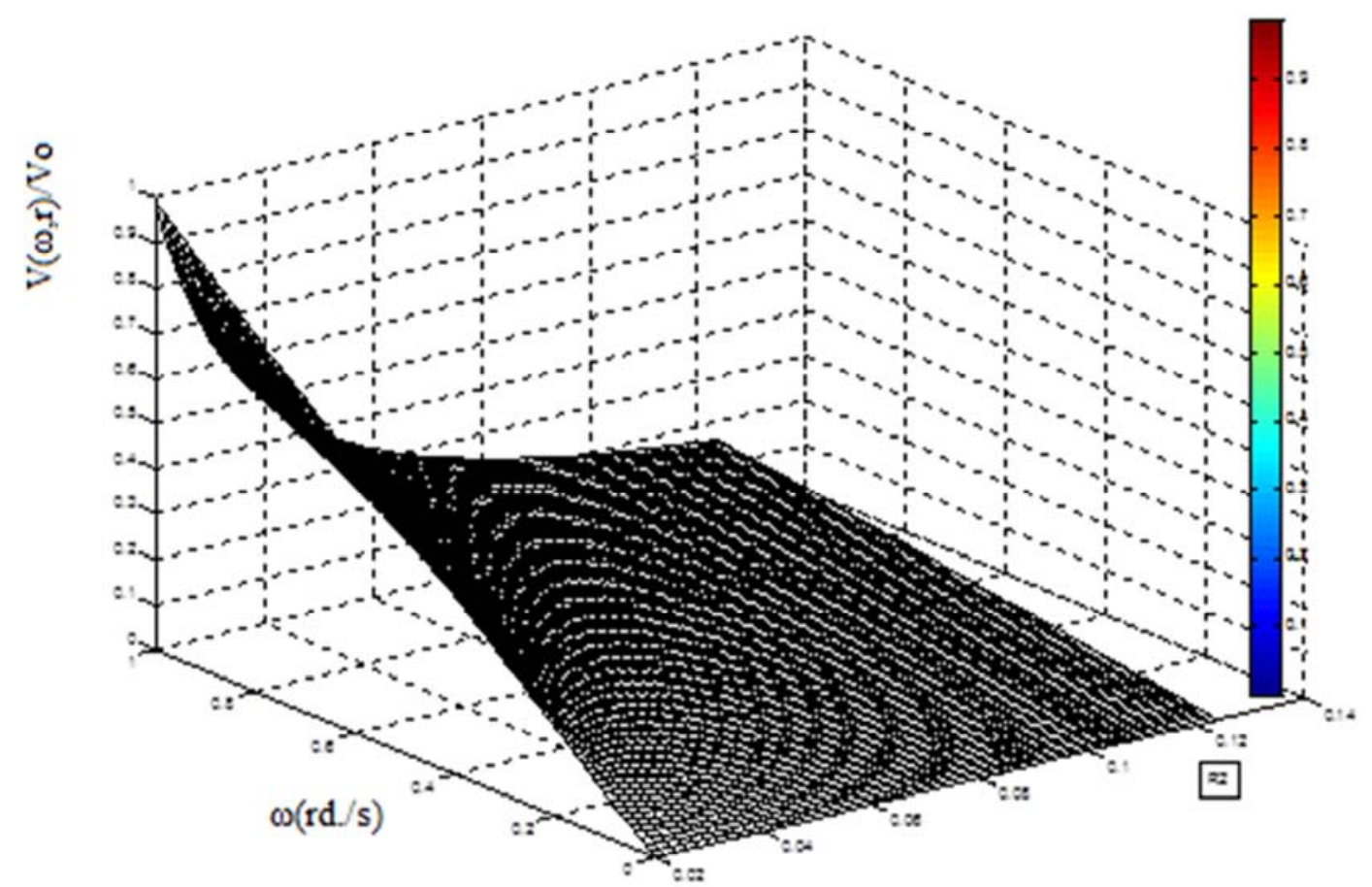

$\mathrm{r}(\mathrm{mm})$

b) 


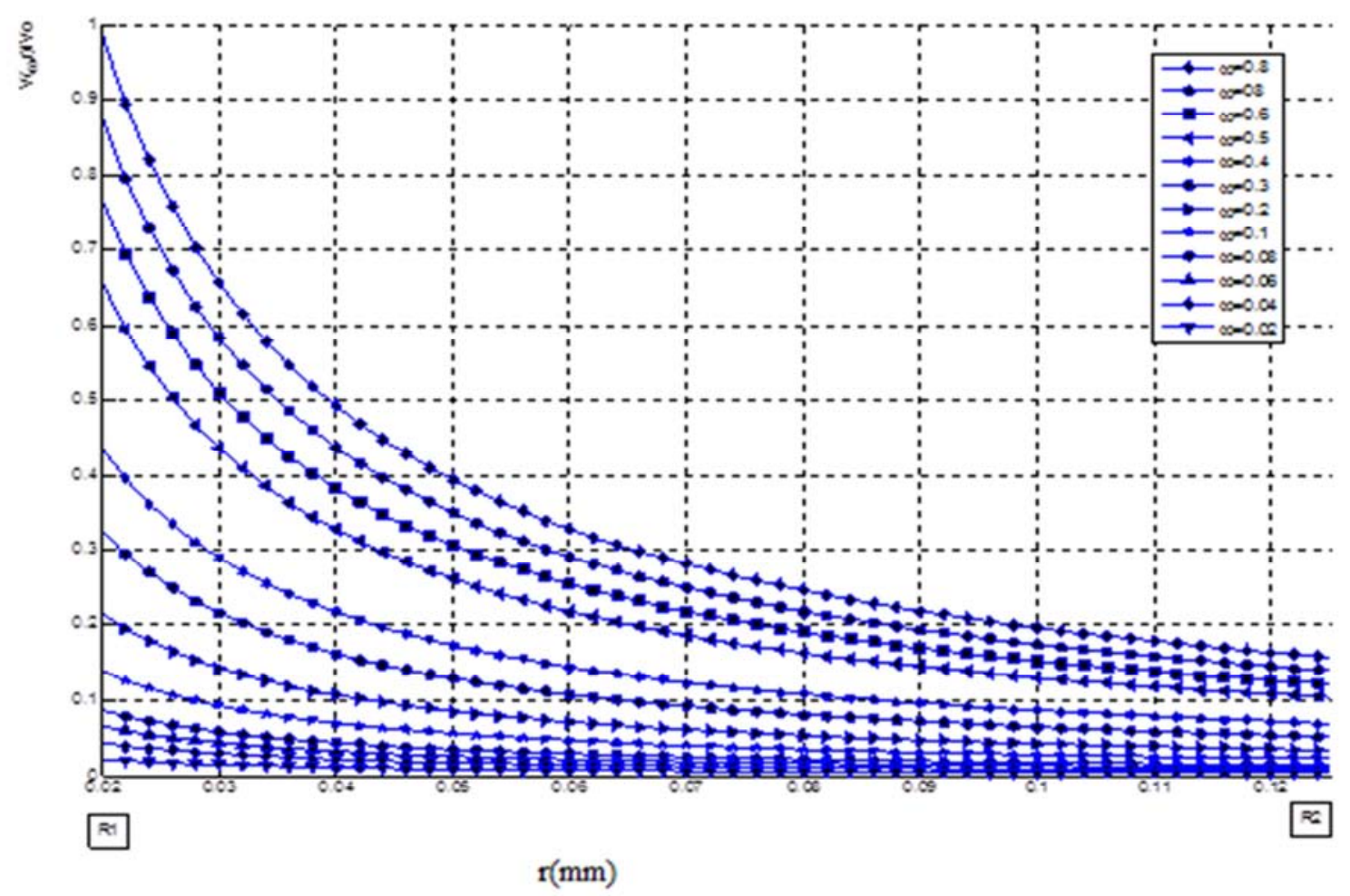

c)

Figure 4. Curves of flow of a solution containing polyethylene-oxide (PEO) of molar mass $106\left(\mathrm{gmol}^{-1}\right)$ and of concentration 1,8\%wt, the angular velocity vary from $(0,02$ to 0,9$) \mathrm{rd} . / \mathrm{s}$ in a going air-gap from $(0,02$ to 0,125$) \mathrm{mm}$. The flow of the fluid is not homogeneous starting from $\omega \mathrm{c} \geq 0,03 \mathrm{rd} / \mathrm{s}$ a) and b) modeling in $3 d$, c) modeling in $2 d$.

b) The angular velocity varies from (0 to 1$) \mathrm{rd} . / \mathrm{s}$ in a going air-gap from $(0,02$ to 0,04$) \mathrm{mm}, \omega \mathrm{c} \geq 0,01 \mathrm{rd} / \mathrm{s}$

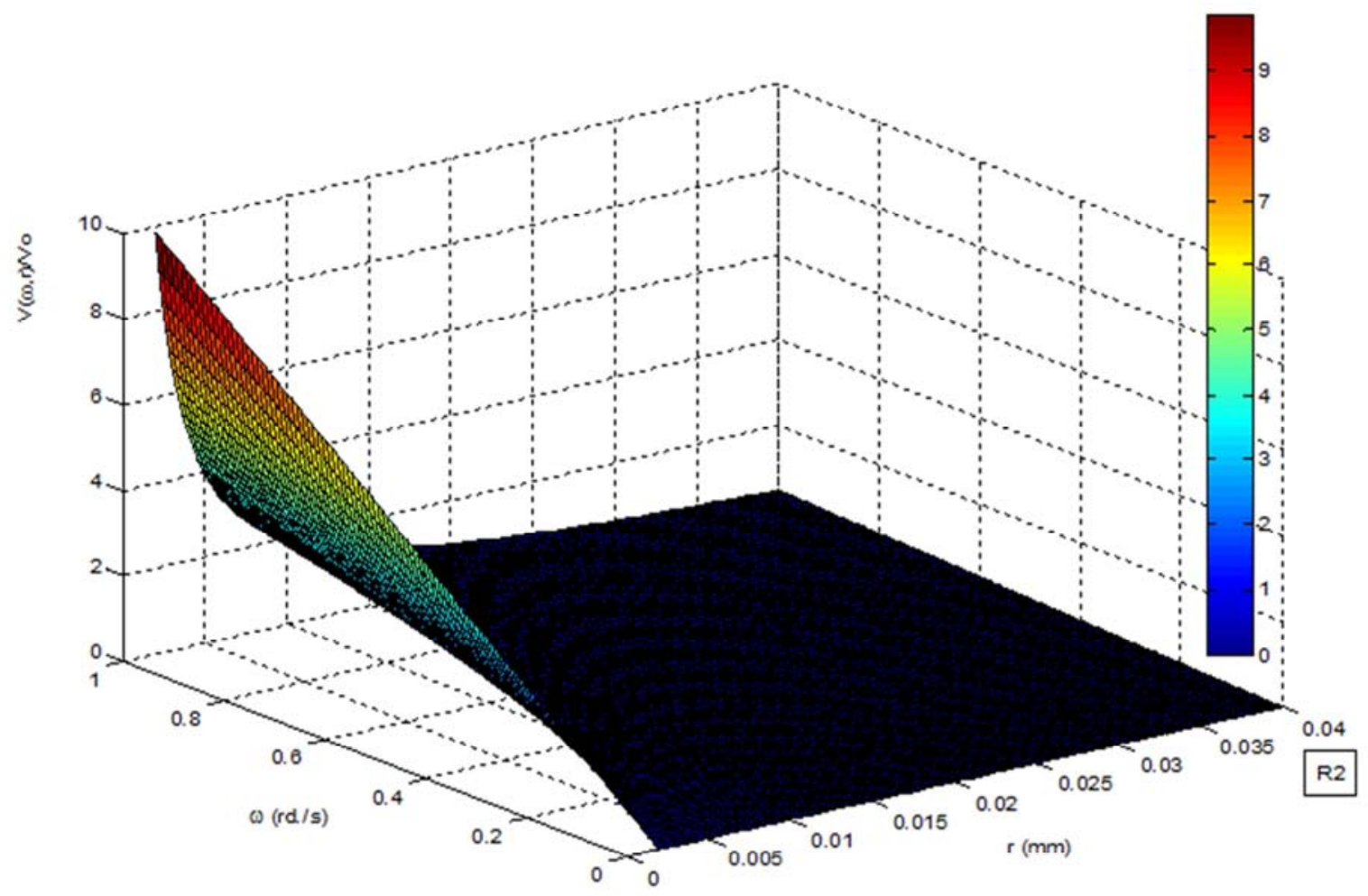




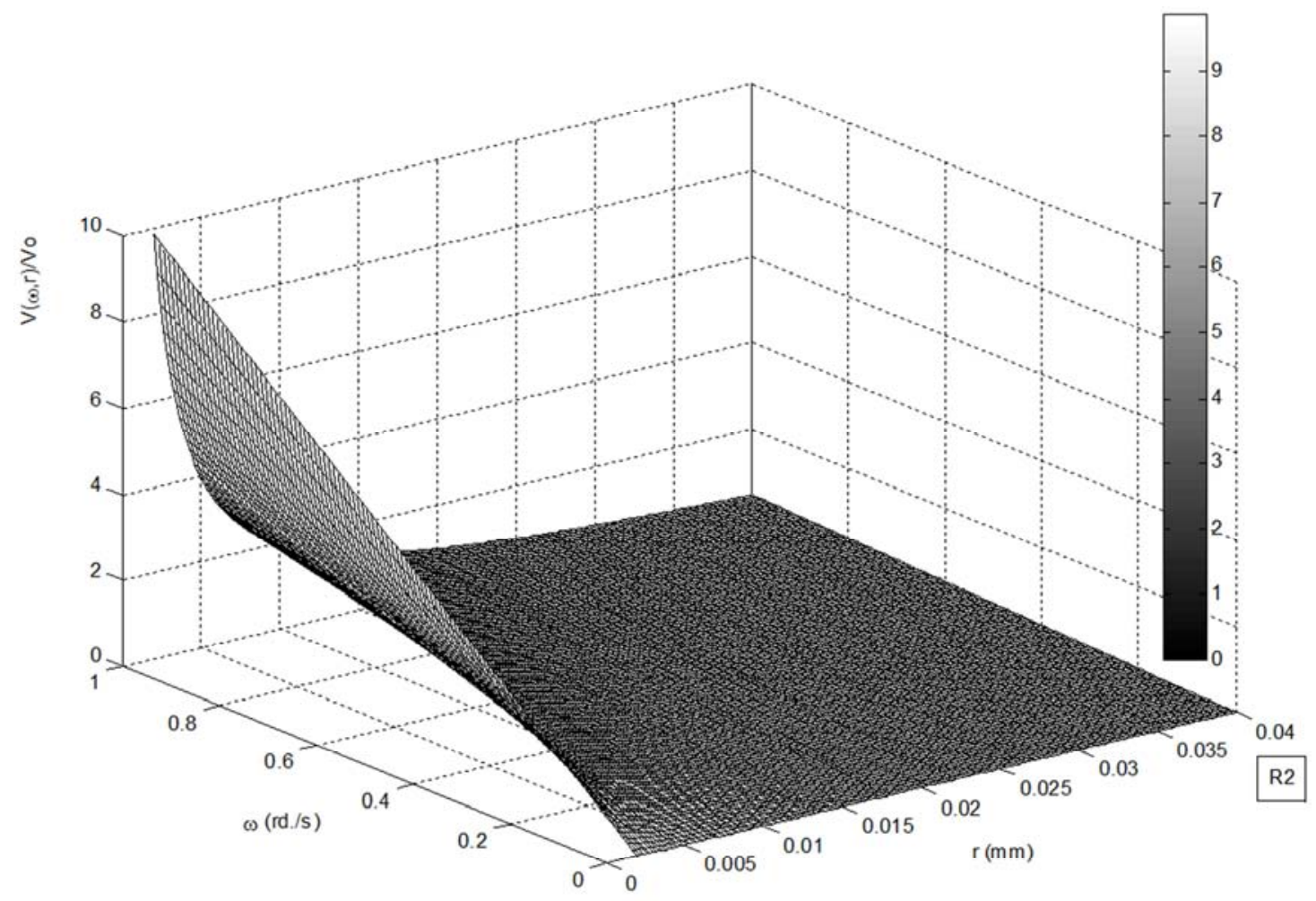

R1

b)

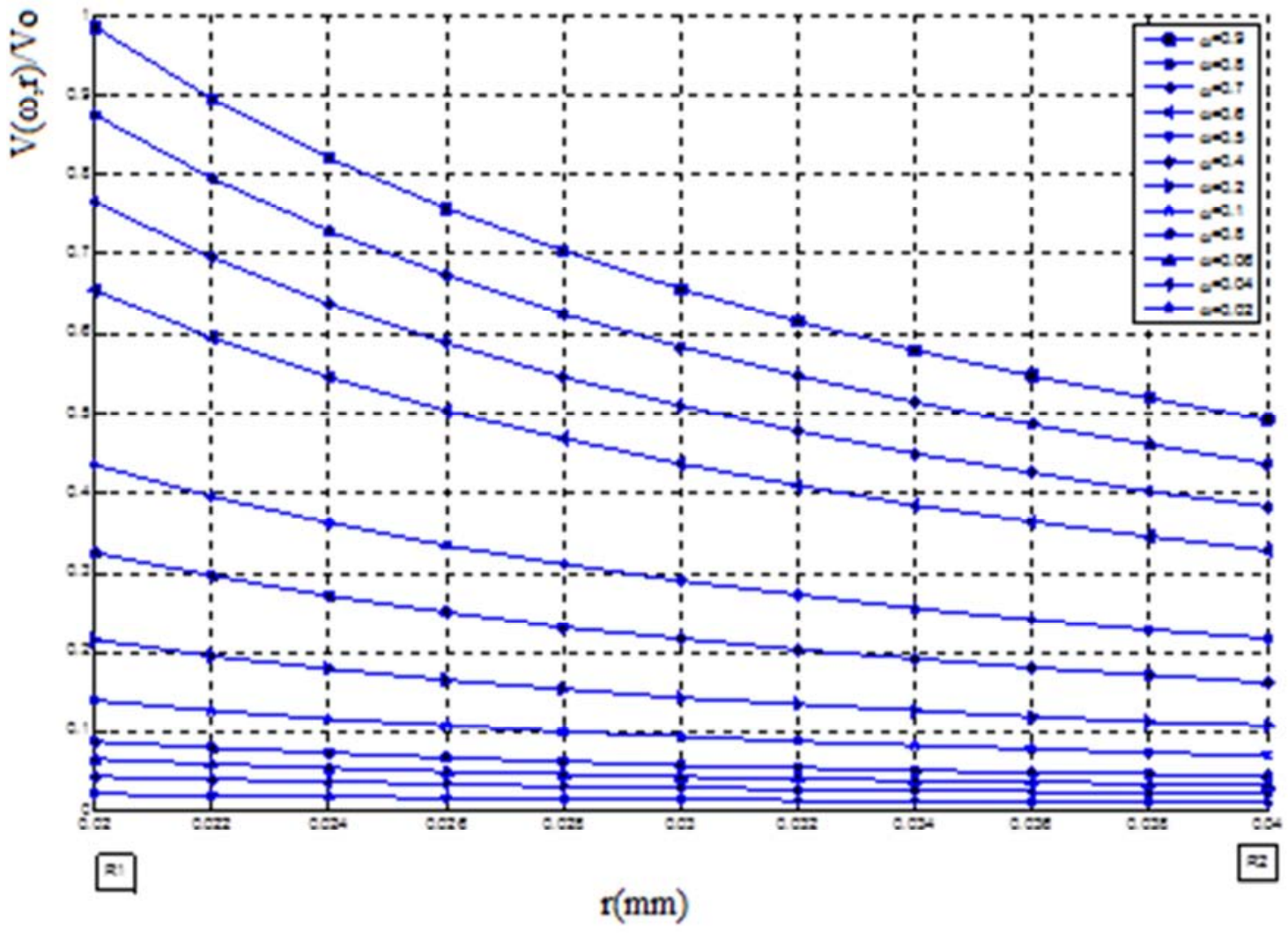

c)

Figure 5. Curves of flow of a solution containing polyethylene-oxide (PEO) of molar mass $10^{6} \mathrm{gmol}^{-1}$ and of concentration 1,8\% wt, the angular velocity vary from $(0,02$ to 0,9$) \mathrm{rd}$./s in a going air-gap from $(0,02$ to 0,04$) \mathrm{mm}$. The flow of the fluid is not homogeneous starting from $\omega_{C} \geq 0,1 \mathrm{rd}$. $/ \mathrm{s}$ a) and b) modeling in $3 D$, c) modeling in $2 D$. 
c) The angular velocity varies from $(0,02$ to 0,9$) \mathrm{rd}$./s in a going air-gap from $(0,02$ to 0,028$) \mathrm{mm}, \omega_{\mathrm{C}} \geq 0,2 \mathrm{rd}$./s.

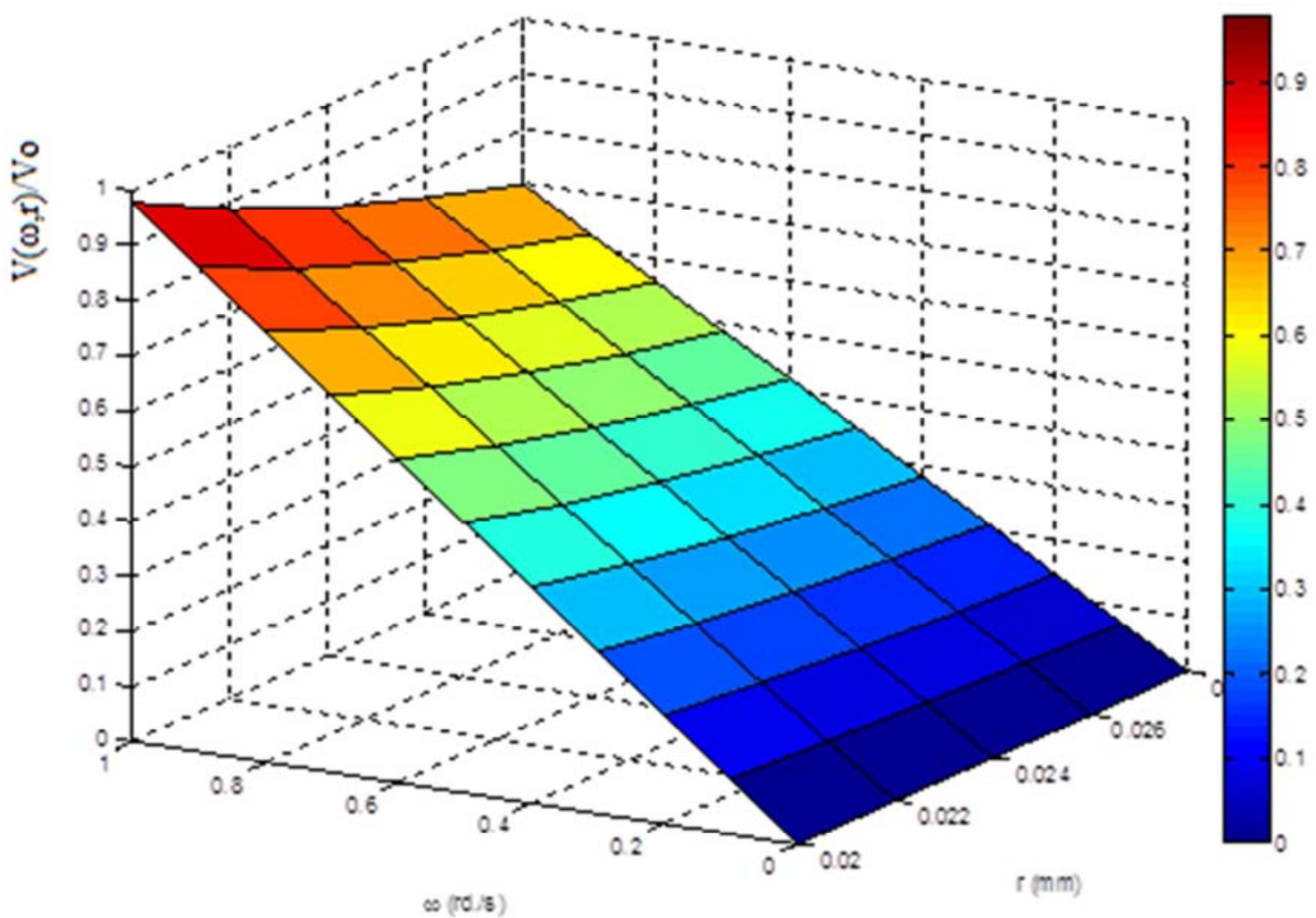

a)

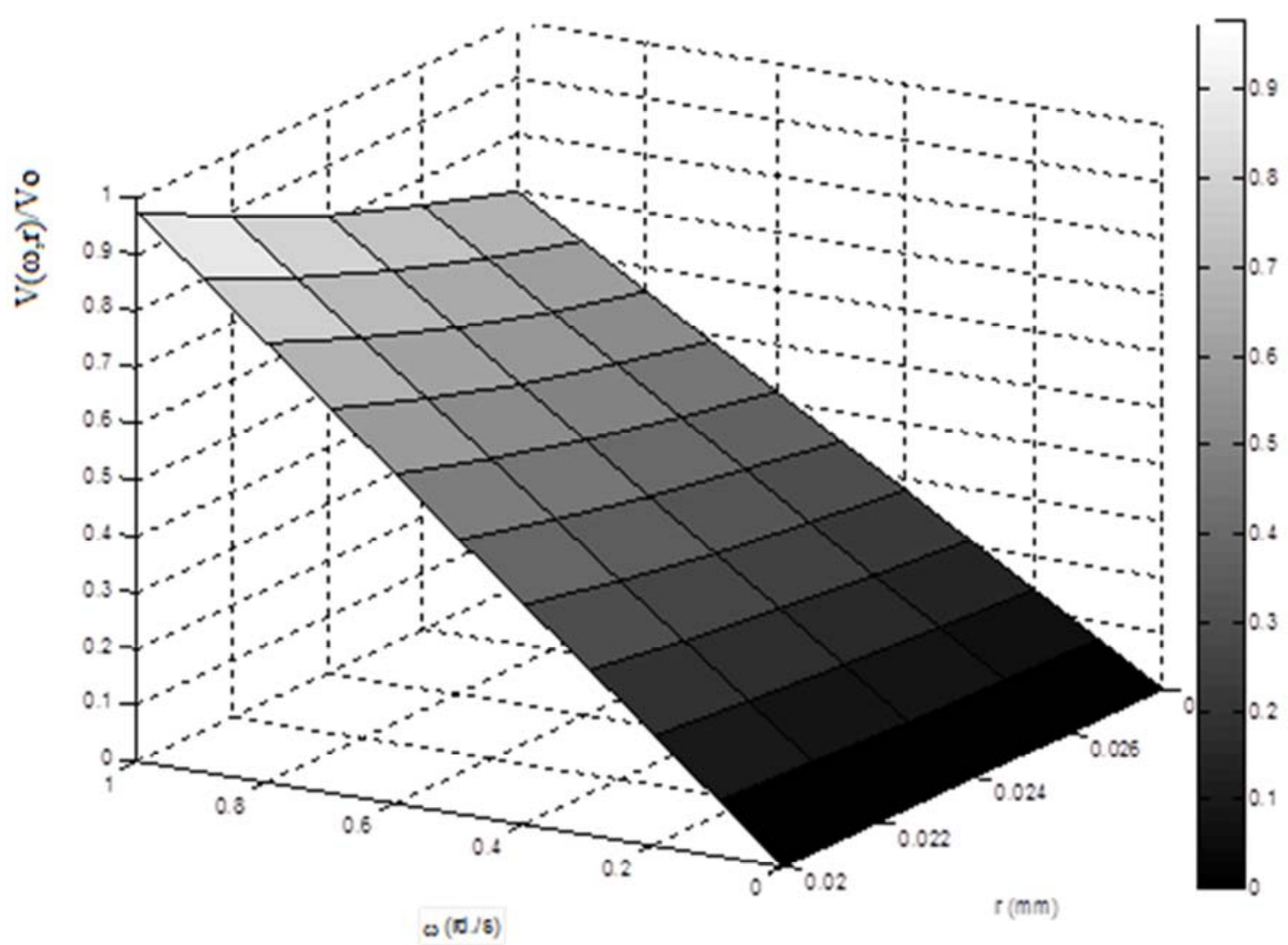




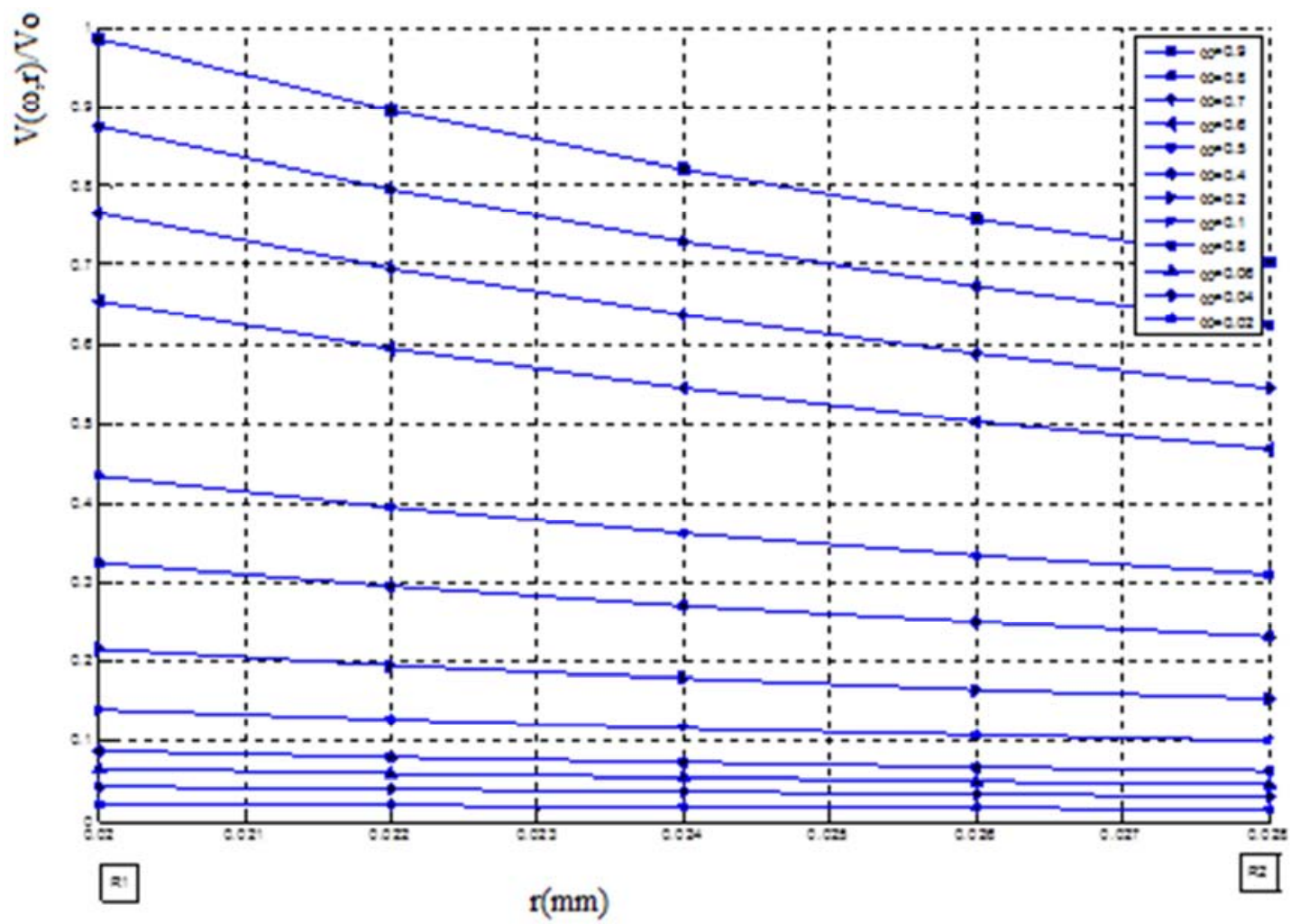

c)

Figure 6. Curves of flow of a solution containing polyethylene-oxide (PEO) of molar mass $10^{6}$ gmol $^{-1}$ and of concentration 1,8\%wt, the angular velocity vary from $(0,02$ to 0,9$) \mathrm{rd}$./s in a going air-gap from $(0,02$ to 0,028$) \mathrm{mm}$. The flow of the fluid is not homogeneous starting from $\left.\omega_{c} \geq 0,2 \mathrm{rd} . / \mathrm{s} a\right)$ and $\left.\mathrm{b}\right) \mathrm{modeling}$ in $3 D$, c) modeling in $2 D$.

d) The angular velocity varies from ( 0 to 1$) \mathrm{rd} . / \mathrm{s}$ in a going air-gap from $(0,02$ to 0,022$) \mathrm{mm}$. $\omega_{\mathrm{c}}=0,9 \mathrm{rd} . / \mathrm{s}$.

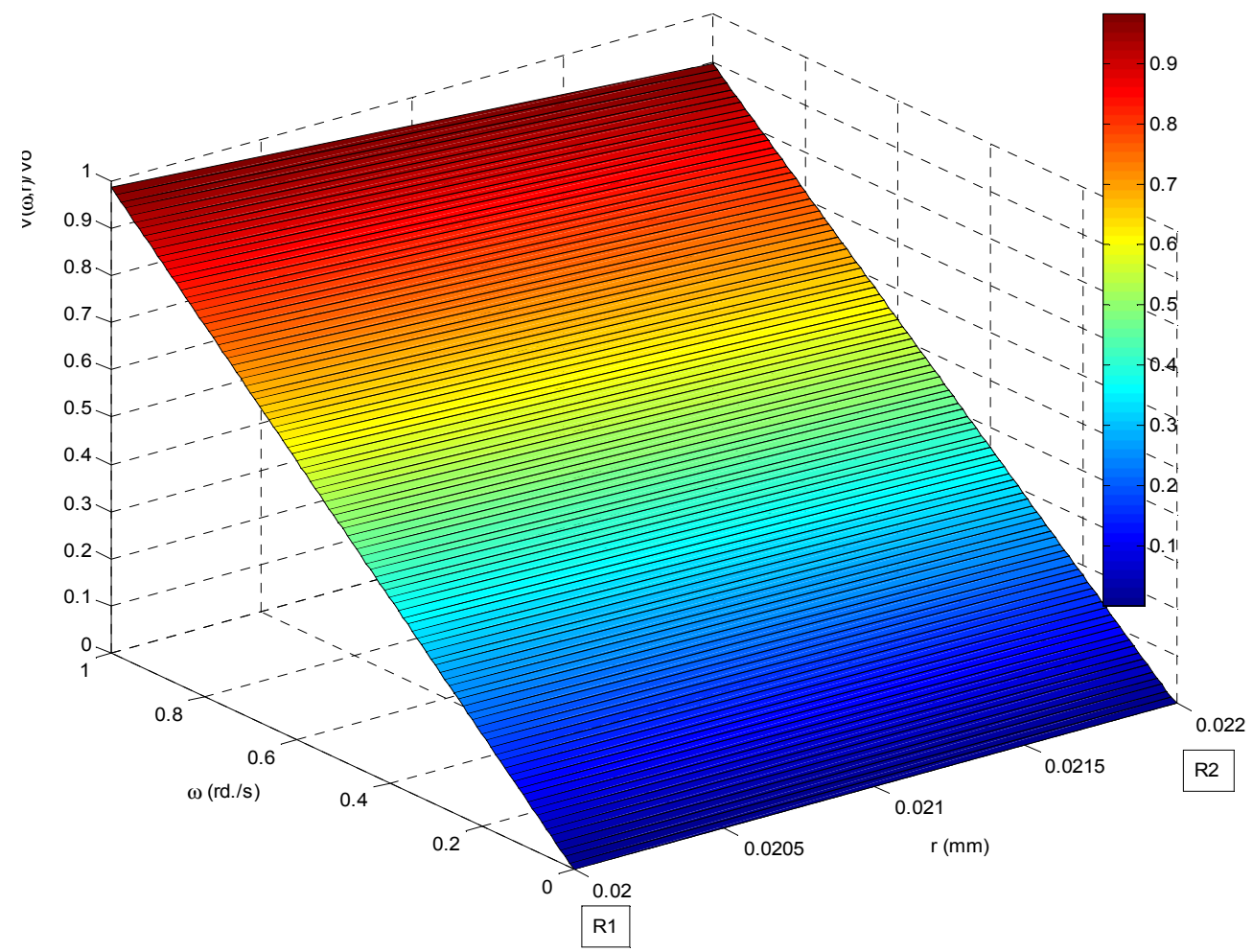




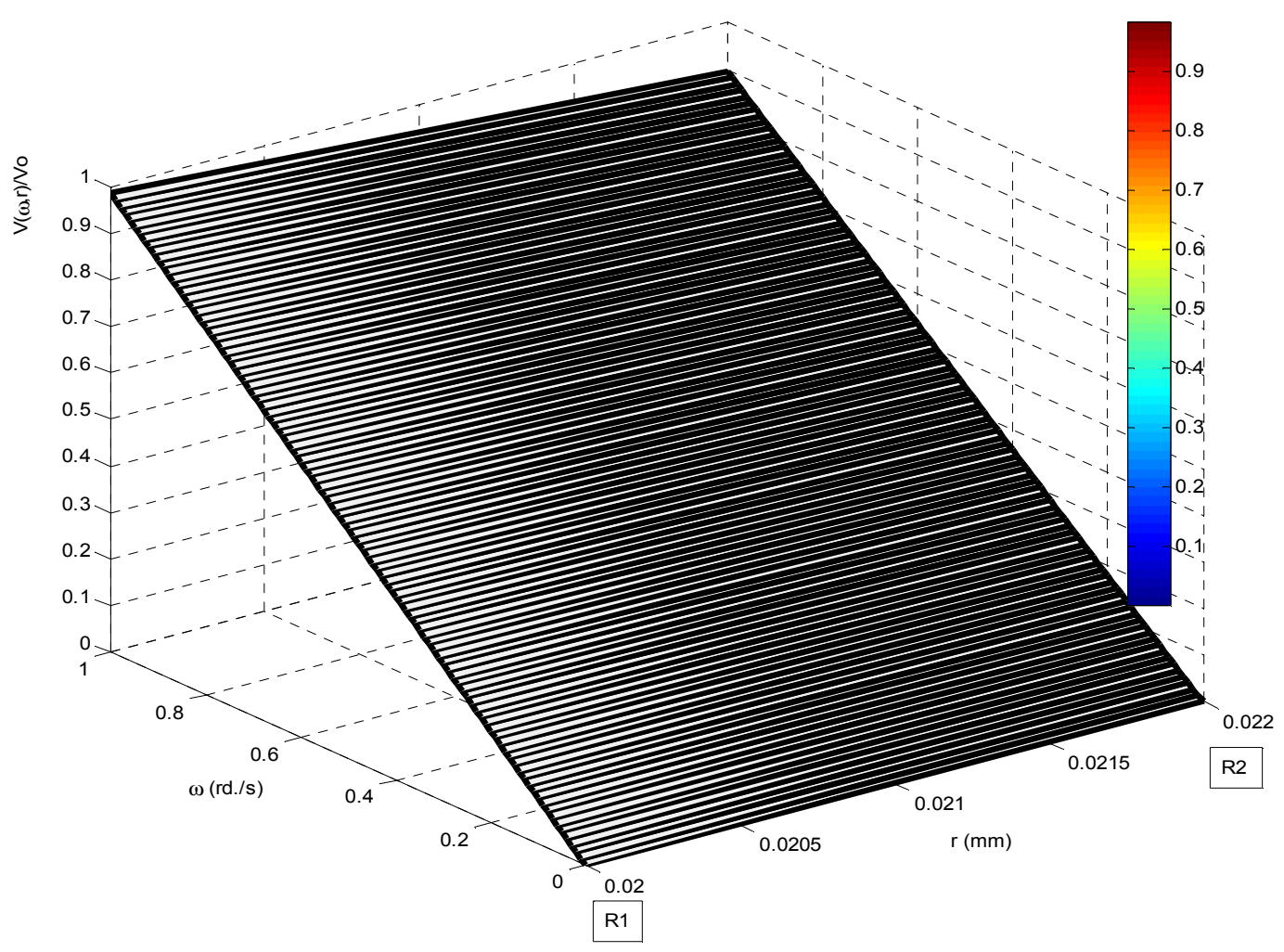

b)

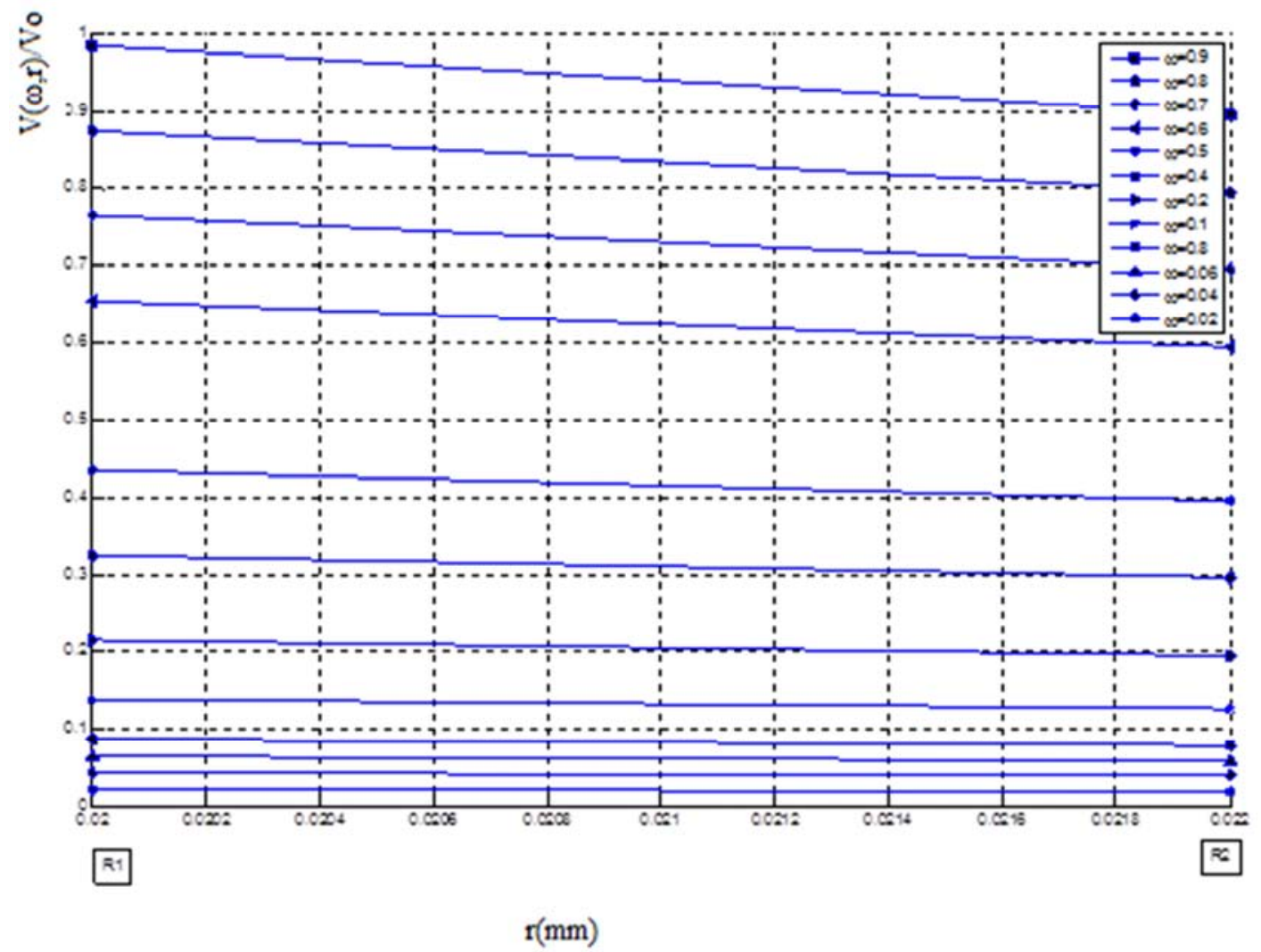

c)

Figure 7. Curves of flow of a solution containing polyethylene-oxide (PEO) of molar mass $10^{6} \mathrm{gmol}^{-1}$ and of concentration $1,8 \%$ wt, the angular velocity vary from $(0,02$ to 0,9) $\mathrm{rd} . / \mathrm{s}$ in a going air-gap from $(0,02$ to 0,022) $\mathrm{mm}$. The flow of the fluid is completely homogeneous for all the values of $\omega \mathrm{rd} . / \mathrm{s}:$ a) and b) modeling in $3 D, c)$ modeling in $2 D$ the air-gap of $0,022 \mathrm{~mm}$ is appropriate for rheological measurements of this structure. 


\section{Conclusion}

The curves of flow of the solution of polyethylene oxide in the cylindrical geometry of Couette are characterized by a heterogeneous behavior, influenced initially by the properties of this solution, then by the geometry of the cylinder and the experimental conditions. These characteristics are found in measurements of these profiles speeds deployed in the device of Couette whose dimension of $0,022 \mathrm{~mm}$ is the higher limit of this geometry for a uniform flow. It is at the same time the lower limit of the opposite problem, i.e. dimension from which begins a nonuniform flow. Under the same conditions of geometry, the angular velocity is a factor of progressive homogenisation of the flow of the structure in all the air-gap: interior cylinder towards the cylinder external and of the values higher lower than the values. This method suggested to characterize the curve of flow of the fluid in shearing in the geometry of Couette is simple and effective. However, the numerical results obtained showed its relevance which makes it possible to be freed from certain limitations related to this type of test: cut too small air-gap, choice a priori of a law of behavior, mode of flow in the air-gap, preliminary evaluation by another procedure of the threshold of flow.

\section{References}

[1] G. Ovarlez, S. Rodts, X. Chateau and P. Coussot (2009). Phenomenology and physical origin of shear-localization and shear-banding in complex fluids, Rheol. Acta, 48, 831-844.

[2] E Guyon, J. P. Hulin and L Petit. (2001). Hydrodynamic physics. EDP Sciences/CNRS Editions, Paris.

[3] G. Couarraze and J L Grossior. (2000). Initiation of rheology. 3rd edition. Lavoisier, Cachan Cedex-France.

[4] C. Ancey (2005). Solving the Couette inverse problem using a wavelet-vaguelette decomposition. J. Rheology, 49, (2), 441460.

[5] N. Ngarmoundou, R. G. Ousman, B. Mahamat and A. C. Beye. (2017). Flow curves in the centered cylindrical Couette geometry of polyethylene oxide solution. polyethylene oxide solution. Open Journal of Fluids Dynamics, 7, (4), 673-695.

[6] J. F. Berret. (2006), Rhéology of worwlike micelles: equilibrium properties and shear-banding transitions. Molecular Gels, 6, 667-720.

[7] G. Porte, J. F. Berret and J. J. Harden. (1997). Inhomogenous flows of complex fluids:Méchanical instability versus non equilibrium phase transition. Europ. Phys. Journal E, 7, 459472 .

[8] N. A. Spenley, M. E. Cates and T. C. Meleiah, (1993). Nonlinear rheology of wormlikes micelles, Phys. Rev. Lett., 71, (6), 939-942.
[9] Huang, N. (2005). Rheology of the granular pastes. Thesis of Doctorate of the University Paris 6-Physics of the liquids.

[10] Fall, A. (2008). Rhéophysique of the complex fluids: Flow and Blocking of concentrated suspensions. Thesis of Doctorate of the University of Paris 7 macroscopic physics.

[11] Berret, J. F (2006). Rhéology of worwlike micelles: equilibrium properties and shear-banding transitions. Molecular Gels, 6, 667-720,

[12] Porte, G., Berret, J. F. Harden, J. (1997). Inhomogenous flows of complex fluids: Méchanical instability versus non equilibrium phase transition. Europ. Phys. [E-Journal], 7, 459-472.

[13] Spenley, N. A., Cates, M. E., Meleiah, T. C. (1993). Nonlinear rheology of wormlikes micelles. Phys. Rev. Lett., 71(6): 939942 .

[14] Bruneau, C H., Gay, C, Hake, T. (2010). Bands of shearing in a continuous model of foam or concentrated emulsion: How a homogeneous material with $3 d$ can seem inhomogenous with $2 d$.

[15] Koblan, W. E., Abdel B., Karim B. Use of the model for the rheological characterization of polymer solutions. Discussion of parameters, 44th Annual Colloquium of the French Group of Rheology, Strasbourg 4, 5 and 6 November 2009.

[16] Riahi, M., Ouazzani, T., Skali Lami, S. (2017). Rheological characterization of an aqueous solution of Polyethylene-Oxide in different concentrations, 13th Congress of Mechanics, Meknes-Morocco from 11 to 14 April 2017.

[17] Barnes, H. A. (1999). The yield stress-a review or ' $\pi \alpha v \tau \alpha \rho \varepsilon \iota^{\prime}-$ everything flow. Journal of Non-Newtonian Fluids Mechanics. 81, 133-178.

[18] Cheng, D. C-H. (1985). Yield stress, a time dependant property and how to measure it. Rheologica Acta, 25, 542554.

[19] Picard, G. (2004). Heterogeneity of the flow of the threshold fluid: phenomenological approach and elastoplastic modeling, $\mathrm{PhD}$ thesis. University of Paris VII. Denis-Dederot.

[20] Schurz, J. (1990). The yield stress - an ampirical reality. Rheological Acta, 29. 170-171.

[21] Perge, C., Fardina, M. A., Divouxb, T., Taberleta, N., Mannevillea, S. (2013). Complex fluids under shear: some instabilities with nul Reynolds number, 21st French Congress of Mechanics Bordeaux, 26 to 30 August 2013.

[22] Lami, S., Leclerc, S., Mathieu, J., Quezennec, C., Guerrin, D. (2015). Rheology and suspension flow of nano-fiber cellulose fibers MRI investigation in a Couette device, 12th Mechanics Congress, Casablanca-Morocco from 21-24 April 2015.

[23] Rigal, C. (2012). Behavior of c omplex fluids under flow: Experimental approach by nuclear magnetic resonance and optical technique and numerical simulations, Thesis of the University of Lorraine, Mechanics and Energetics. 